University of South Carolina

Scholar Commons

6-1999

\title{
Dynamics of Formation of Soliton Conductivity in a 2 D-array of Linear Chains Containing Commensurate Charge Density Waves Near the Contact with a Normal Metal
}

Yuriy V. Pershin Dr

University of South Carolina - Columbia, pershin@physics.sc.edu

Alexander S. Rozhavsky

Follow this and additional works at: https://scholarcommons.sc.edu/phys_facpub

Part of the Physics Commons

\section{Publication Info}

Published in Low Temperature Physics, Volume 25, Issue 6, 1999, pages 453-458.

Pershin, Y. V., \& Rozhavsky, A. S. $(1999,2001)$. Dynamics of formation of soliton conductivity in a 2 D-array of linear chains containing commensurate charge density waves near the contact with a normal metal.

Low Temperature Physics, 25(6), 453-458.

(C) Low Temperature Physics, 1999, 2001, American Institute of Physics

http://ltp.aip.org/resource/1/ltpheg/v25/i6/p453_s1?isAuthorized=no

This Article is brought to you by the Physics and Astronomy, Department of at Scholar Commons. It has been accepted for inclusion in Faculty Publications by an authorized administrator of Scholar Commons. For more information, please contact digres@mailbox.sc.edu. 


\title{
Dynamics of formation of soliton conductivity in a $2 D$-array of linear chains containing commensurate charge density waves near the contact with a normal metal
}

\author{
Yurij V. Pershin and Alexander S. Rozhavsky \\ B. Verkin Institute for Low Temperature Physics and Engineering, 47 Lenin Avenue, 310164, Kharkov, \\ Ukraine* \\ (Submitted January 18, 1999; revised February 4, 1999) \\ Fiz. Nizk. Temp. 25, 609-615 (June 1999)

\begin{abstract}
We make a numerical study of the conversion of conduction electrons into charge density wave (CDW) topological solitons at the interface between a normal metal and a $2 D$-array of the $\mathrm{CDW}$-carrying linear chains. The interplay of commensurability potential, interchain interaction, and electric field on the dynamics of soliton formation is studied. When the interchain interaction exceeds the commensurability energy, the dynamic mechanism of creation of fractionally charged solitons near the contact is suppressed and specific contact nonlinearity in transport current is not observed. (C) 1999 American Institute of Physics.
\end{abstract} \\ [S1063-777X(99)01106-8]
}

\section{INTRODUCTION}

Some quasi-one-dimensional metallic alloys undergo phase transition to the Peierls dielectric (PD) state at low temperatures (see, e.g., ${ }^{1-3}$ ). PD is characterized by a complex order parameter $\Delta \exp (i \varphi)$, where $\Delta$ is the gap in a single-electron spectrum and phase gradients define the collective charge transfer: CDW conductivity. Topologically stable nonlinear phase excitations, in particular, solitons and antisolitons, serve as the elementary CDW charge carriers. The soliton description is more or less successful in explanation of the nonlinear bulk transport (see, e.g., the Reviews 1 and 2). However, one principal aspect of CDW-physics, viz, the problem of interaction of the current-carrying CDWphase deformations with conduction electrons, in particular, the nature of $\mathrm{CDW} /$ metal electrode interface phenomena, is not yet entirely understood and controversial explanations still arises.

To describe the process of charge transformation at the $\mathrm{CDW} /$ normal metal interface, the ideology of phase slip centers (PCS) which exploits the analogy between the PSC and dislocations had been put forward (see, e.g., Refs. 4-7). The physics behind the PSC is the strongly pronounced polaron effect: ${ }^{3}$ i.e., conduction electrons imbedded in the conduction band of a quasi-one-dimensional semi-conductor are unstable against self-trapping and subsequent absorption by the valence band where they are finally converted into CDW phase solitons. ${ }^{9}$ Interchain interaction provides aggregation of solitons into dislocation-like loops: PSCs. Charge transformation takes place near the contact with a normal metal. In the cited publications ${ }^{4-7}$ (see also references therein), the PSCs were treated as the static objects.

Dynamics of conversion was studied in a series of pioneer papers. ${ }^{8-10}$ It was shown that prior to formation of PSC conduction solitons manifest highly nontrivial individual behavior, and the proper hierarchy of time scales which governs the charge transformation was established. Self-trapping is connected with local gap deformations in conducting chains. The potential barrier for the self-trapping is $\sim \Delta$, and during the time $\sim \hbar / \Delta$ the quasiparticles spectrum is matched to a local value $\Delta(\mathbf{r}, t)$; the time of the gap deformation is of order of $\bar{\omega}^{-1}(\bar{\omega}$ is the frequency of the Peierls phonons which is of the order of the Debye frequency $\left.{ }^{2,3}\right)$, the interchain interaction being of order $T_{c}\left(T_{c}\right.$ is the temperature of the Peierls transition), it defines the time $\hbar / T_{c}$ of the interchain phase coherence onset. In a weakly coupled array of highly conducting chains, when $T_{c} \ll \hbar \bar{\omega} \ll \Delta$, the selftrapping of electrons occurs in individual chains independently ${ }^{3,4}$ and the charge transformations proceeds in two steps, each characterized by its own time: transfer of conduction electrons into the valence band in a single chain at $\tau_{\Delta} \sim \bar{\omega}^{-1}$, and formation of a collective charge carrier in this chain at $\tau_{\varphi} \gg \tau_{\Delta}$. The time $\tau_{\varphi}$ is the intrinsic scale of the CDW-phase Hamiltonian, ${ }^{2-4}$ at $t<\tau_{\varphi}$ individual charge carriers obey the Lagrange equations supplemented by the boundary conditions. The latters are formed during the time $t<\tau_{\Delta} ; ;^{7}$ the initial phase perturbation is localized near the interface over the distance of the order of $V_{F} \tau_{\Delta} \sim \xi_{0}$ $=\hbar V_{F} / \Delta$; where $\xi_{0}$ is the amplitude coherence length in PD. The jump $\delta \varphi$ of the initial phase profile $\varphi(t=0)$ is defined by the charge conservation law in the process of selftrapping. Indeed, the collective CDW charge density $\rho$ in a single chain is related to phase gradient via the Fröhlich relation:

$$
\rho=\frac{e}{\pi} \frac{\partial \varphi}{\partial x}
$$

When $q$ electrons are converted into the CDWcondensate, the phase acquires a local deformation with the net phase shift:

$$
\delta \varphi=\varphi(x=\infty)-\varphi(x=-\infty)=q \pi .
$$

It was shown in ${ }^{9}$ that during the elementary act of selftrapping at the metal/PD interface the charge $2 e$ is transformed into a $\mathrm{CDW}$ in a single chain, i.e. two electrons with 
opposite spins are self localized during the time $\bar{\omega}^{-1}$. This process resembles the Andreev reflection in superconductors. Thus, the initial condition to the phase equations of motion leads to $q=-2$ in Eq. (2). As the scale $\xi_{0}$ is much less than any intrinsic length in the phase Hamiltonian, ${ }^{2-4}$ we can formulate the initial condition as a point-like step function with the height equal to $-2 \pi$. $^{7}$

In our previous publications ${ }^{8,10}$ we have studied the evolution of the initial CDW profile both analytically and numerically in two models, when self-trapping occurs:

1) in a central chain which belongs to a cluster of nearest chains containing an incommensurate CDW. The electric field was not taken into account ${ }^{8}$

2) in an isolated chain containing commensurate CDW in the presence of a dc-electric field. ${ }^{10}$

It was shown that the initial condition always transforms into stable topological Sine-Gordon (SG) solitons. In the model of nearest chains cluster each soliton has charge $2 e$, and the role of the bulk term in the SG equation plays the role of interaction between chains of the type $\sin \left(\varphi_{0}-\varphi_{1}\right)$, where $\varphi_{0}$ and $\varphi_{1}$ are the phases in the central and nearest chains. In a commensurate CDW we have observed the effect of a charge fractionalization (see, e.g., Refs. 1 and 2) when the initial profile decays into $M$ fractionally charged solitons (an integer $M>2$ is an index of commensurability) each carrying charge:

$$
q_{s}=2 e / M \text {. }
$$

It was shown in Ref. 10 that soliton-antisoliton pairs with charges $\pm q_{s}$ (3) are created in a dc-electric field from an initial CDW profile, thus giving rise to an additional contact non-linearity in the CDW-conductivity.

It is certainly interesting to study the evolution of initial CDW profile and interaction of phases in different chains in a more general $2 D$ model, which takes into account the effects of commensurability, inter-chain interaction and electric field. This problem is not integrable; it is solved numerically in this paper.

\section{MODEL}

Consider the $2 D$-array of the $\mathrm{CDW}$-containing chains which occupy the semi-axis $x \geqslant 0$. The Lagrangian of the system is (see, e.g., Ref. 2):

$$
\begin{aligned}
L= & \frac{1}{\pi \hbar V_{F}} \sum_{i}\left[\frac{\Delta^{2}}{\bar{\omega}^{2}}\left(\frac{\partial \varphi_{i}}{\partial t}\right)^{2}-\frac{\hbar^{2} V_{F}^{2}}{4}\left(\frac{\partial \varphi_{i}}{\partial x}\right)^{2}\right. \\
& +\frac{\Delta^{2}}{\bar{\omega}^{2}} \frac{2}{M^{2}} \omega_{0}^{2} \cos M \varphi_{i}+2 T_{c}^{2} \cos \left(\varphi_{i}-\varphi_{i-1}\right) \\
& \left.+\frac{e}{\pi} \hbar V_{F} E\left(\varphi_{i}-\widetilde{\varphi}\right)\right]
\end{aligned}
$$

where $\varphi_{i}$ denotes the phase in the $i$-th chain, $\omega_{0}$ is the commensurability frequency, and $\widetilde{\varphi}$ is the phase at $x \rightarrow+\infty$. Such form of the last term in Eq. (4) takes into account the renormalization of the phase in each chain in the presence of an electric field. form:

The equation of motion for the Lagrangian (4) has the

$$
\begin{aligned}
& \frac{\partial^{2} \chi_{i}}{\partial t^{2}}-\frac{\partial^{2} \chi_{i}}{\partial y^{2}}+A \sin M\left(\chi_{i}+\widetilde{\varphi}\right)+B \sin \left(\chi_{i}-\chi_{i-1}\right) \\
& \quad+B \sin \left(\chi_{i}-\chi_{i+1}\right)=\varepsilon
\end{aligned}
$$

where

$$
\begin{aligned}
& y=\frac{2 \Delta}{\hbar V_{F} \bar{\omega}} x, \quad \varepsilon=\frac{e}{2 \pi} \hbar V_{F} \frac{\bar{\omega}^{2}}{\Delta^{2}} E \\
& A=\frac{\omega_{0}^{2}}{M}, \quad B=\frac{\bar{\omega}^{2}}{\Delta^{2}} T_{c}^{2}, \quad \text { and } \chi_{i}=\varphi_{i}-\widetilde{\varphi} .
\end{aligned}
$$

In a nonzero field $E$ the ground state is:

$$
\widetilde{\varphi}=\arcsin (\varepsilon) .
$$

Equation (7) implies the restriction on the electric field whereby the stable phase configuration exists:

$$
\left|\frac{\varepsilon}{A}\right|<1 \text {. }
$$

Only the fields that obey the condition (8) are considered further.

Equation (5) is supplemented by the initial and boundary conditions which describe conversion of the pair of electrons into a CDW-profile in a central chain $(i=0)$ (see the above discussion of the hierarchy of times in this problem):

$$
\begin{aligned}
& \left.\frac{\partial \chi_{i}}{\partial t}\right|_{t=0}=0, \\
& \chi_{i}(t=0)=-2 \pi \theta\left(\bar{\xi}_{0}-y\right) \delta_{i 0}, \\
& \chi_{i}(y=0)=-2 \pi \delta_{i 0},
\end{aligned}
$$

where $\delta_{i j}=1, i=j$ and $\delta_{i j}=0, i \neq j ; \bar{\xi}_{0}$ is the coherence length $\xi_{0}=\hbar V_{F} / \Delta$ in units (6), $\bar{\xi}_{0} \ll 1$; and $\theta(y)$ is the Heavyside step function.

In what follows, we solve Eq. (5) with the conditions (9) numerically by means of the method of finite differences. The difference equation corresponding to Eq. (5) has the form

$$
\begin{gathered}
\frac{\chi_{i, k+1, l}+\chi_{i, k-1, l}-2 \chi_{i, k, l}}{(\Delta t)^{2}}-\frac{\chi_{i, k, l+1}+\chi_{i, k, l-1}-2 \chi_{i, k, l}}{(\Delta y)^{2}} \\
+A \sin M\left(\chi_{i, k, l}+\varphi_{0}\right)+B \sin \left(\chi_{i, k, l}-\chi_{i+1, k, l}\right) \\
+B \sin \left(\chi_{i, k, l}-\chi_{i-1, k, l}\right)=\varepsilon,
\end{gathered}
$$

where $\Delta t$ is the time step, $\Delta y$ is coordinate step, and $\chi_{i, k, l}$ $=\chi_{i}(\Delta t k, \Delta y l)$. Equation (10) is solved for different values of parameters and at different envelope functions in (9b). The results presented are obtained for $\max (k)=1000(\max (k)$ is the number of sites). It is found in particular that neither changing the shape of the initial perturbation (9b) (rectangular or triangular step) nor increasing the number of sites provides any significant effect on the solution of Eq. (10). 


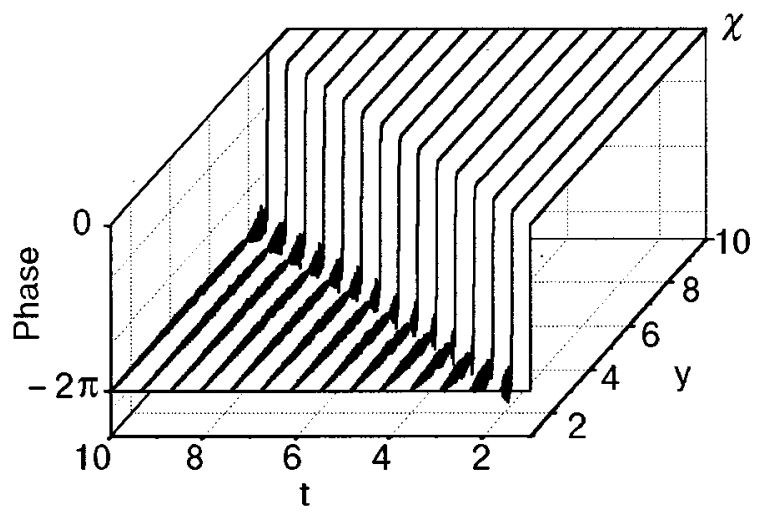

a

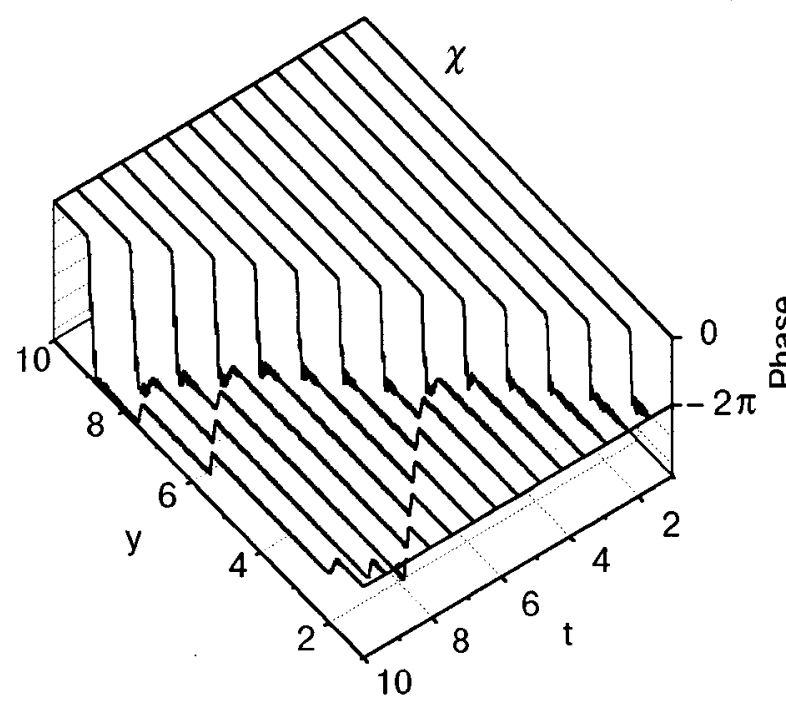

C

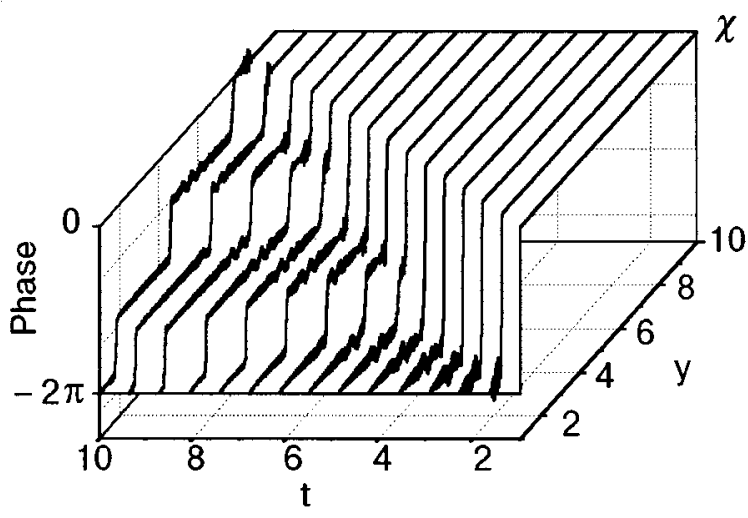

b

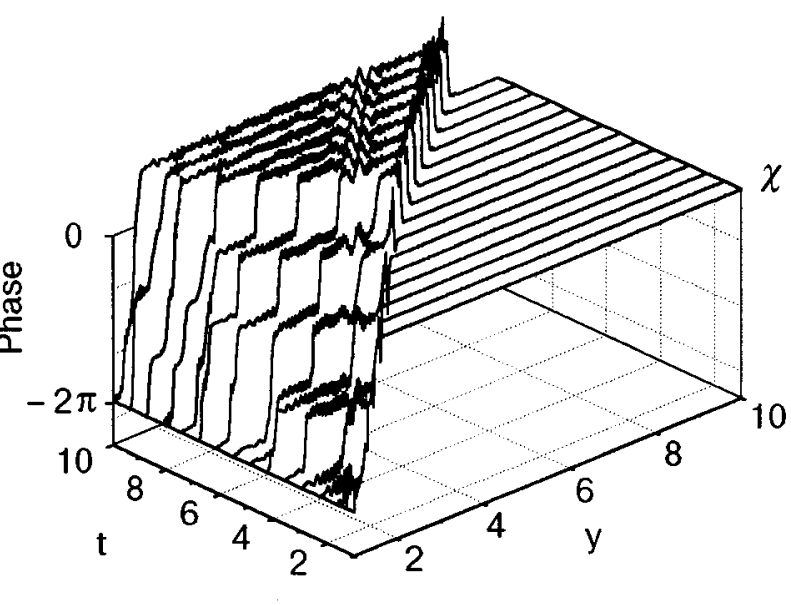

d

FIG. 1. Dynamics of conversion of the electrons to the commensurate one-dimensional CDW. $A=1$ : a-accelerating field: $\varepsilon=0.05$; b-decelerating field: $\varepsilon=-0.05 ; \mathrm{c}$-accelerating field: $\varepsilon=0.2$. The mechanism of the pair creation is observed: $\mathrm{d}$-decelerating field: $\varepsilon=-0.2$. The mechanism of the pair creation is observed.

\section{CONVERSION IN A SINGLE CHAIN}

Dynamics of conversion of conduction electrons into a one-dimensional CDW was studied in. ${ }^{10}$ The system is described by the reduced equation (5):

$$
\frac{\partial^{2} \chi}{\partial t^{2}}-\frac{\partial^{2} \chi}{\partial y^{2}}+A \sin M(\chi+\widetilde{\varphi})=\varepsilon .
$$

In weak decelerating and accelerating fields $(|\varepsilon / A|$ $<0.1)$ the dynamics of phase develops along conventional lines. In an accelerating field the initial profile propagates along the chain keeping safe its " «step-like»" form (9b) (Fig. 1a). In a weakly decelerating field (Fig. 1b), the initial profile loses its stability after some time and splits into commensurability solitons (3), which reverse their direction of motion, and eventually collect near $x=0$. The radiation propagating with the maximum velocity is clearly observed.

The picture changes drastically when $|\varepsilon / A|$ exceeds a threshold field $\varepsilon_{T}$. In this case the charge creation during the evolution of the initial profile is observed both in accelerating (Fig. 1c) and decelerating fields (Fig. 1d). The reversal of the sign of created charges with the change of the field direction unambiguously indicates that the mechanism of the charge formation is the polarization of a CDW vacuum: at $\varepsilon>0$, the soliton $(s)$ and antisoliton $(\bar{s})$ move pro- and contra the electric field correspondingly; at $\varepsilon<0$ soliton and antisoliton change their positions. Such a mechanism of the charge creation is responsible for the specific nonlinearity of the contact conductivity: ${ }^{10}$

$$
j \sim \frac{E \sqrt{E-E_{T}}}{B+\sqrt{E-E_{T}}} .
$$

Note that the threshold field $E_{T}$, which defines the onset of the nonlinear contact conductivity (12), is different from the one normally observed in bulk transport. ${ }^{1,2}$ The latter is of electrostatic origin and of course cannot appear in numerical simulation of Eq. (11). The microscopic origin of the threshold (11) is the concurrence between the energy of solitonantisoliton confinement in a moving phase profile and the electric field, which tends to dissolve the $s \bar{s}$-bound state. ${ }^{10}$ 


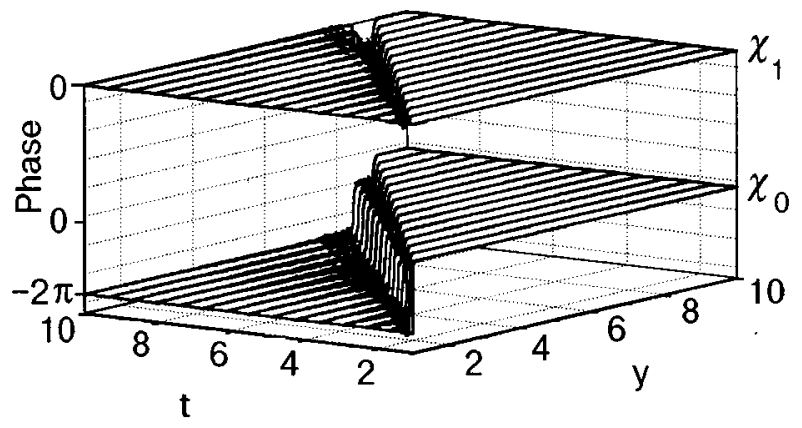

a

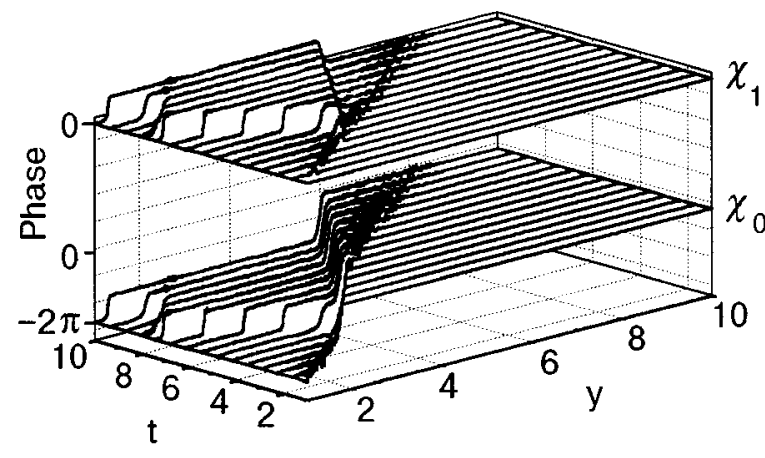

C

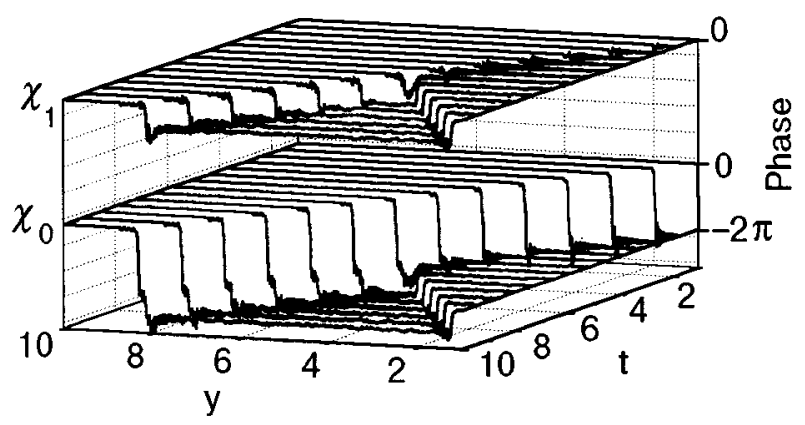

b

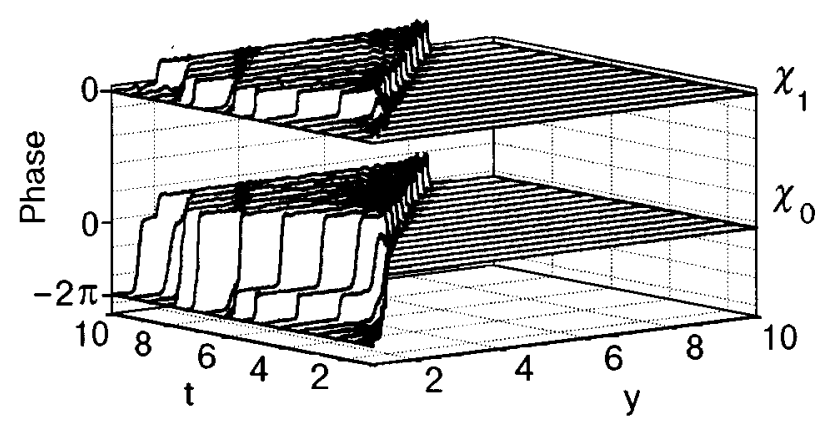

d

FIG. 2. Dynamics of conversion of the electrons to the cluster of the nearest chains. $N=2, B=1$ : a-incommensurate chains: $\varepsilon=0, A=0$. The initial condition transforms to a $2 \pi$-kink surrounded by dipoles; $\mathrm{b}$ - commensurate chains, accelerating field: $\varepsilon=0.4, A=1$. The mechanism of the pair creation is observed; c-commensurate chains, decelerating field: $\varepsilon=-0.2, A=1$. The mechanism of the pair creation is observed; $\mathrm{d}-$ commensurate chains, decelerating field: $\varepsilon=-0.3, A=1$. The mechanism of the pair creation is observed.

\section{CONVERSION IN A 2 D-CLUSTER OF NEAREST CHAINS}

In this model we consider the conversion in a central chain which is surrounded by $N$ symmetrically arranged nearest chains. The symmetry of the problem allows us to describe the dynamics of conversion by the following two equations:

$$
\begin{aligned}
& \frac{\partial^{2} \chi_{0}}{\partial t^{2}}-\frac{\partial^{2} \chi_{0}}{\partial y^{2}}+A \sin M\left(\chi_{0}+\widetilde{\varphi}\right)+N B \sin \left(\chi_{0}-\chi_{1}\right)=\varepsilon, \\
& \frac{\partial^{2} \chi_{1}}{\partial t^{2}}-\frac{\partial^{2} \chi_{1}}{\partial y^{2}}+A \sin M\left(\chi_{1}+\widetilde{\varphi}\right)+B \sin \left(\chi_{1}-\chi_{0}\right)=\varepsilon,
\end{aligned}
$$

where $\chi_{0}$ is the phase in the central chain and $\chi_{1}$ in neighboring ones. Equations (13) are supplemented by the conditions (9). Equations (13) differ from Eq. (11) by the interchain interaction term $B \sin \left(\chi_{0}-\chi_{1}\right)$.

The oversimplified problem (without the electric field and commensurability potential) was solved analytically in Ref. 8. It was shown in Ref. 8 that pair of self-trapped conduction electrons transforms into a charged $2 \pi$-kink localized in a central chain and surrounded by dipoles in neighboring chains. The result of a numerical study of same problem is plotted in Fig. 2a. There are two distinctions between the results obtained analytically and numerically. First is the existence of the radiation (Fig. 2a) which has been dropped in. ${ }^{8}$ Second, decrease of the velocity of the $2 \pi$-kink (Fig. 2a), which is the typical feature of the soliton-type solutions in the discrete Sine-Gordon equation. ${ }^{11,12}$ The inclusion of the commensurability $[A \neq 0$ in Eq. (13)] results in suppression of the charged dipoles.

Taking into account the electric field in the r.h.s. of Eqs. (13) leads to various pictures, which depend on the equation parameters. As in the model of a single chain, the threshold field is observed, which is higher that in the one-dimensional model and which depends on the number of neighboring chains $N$, the parameters $A$ and $B$, and sign of the field. Increasing the number of chains leads to increasing the threshold field. In weak accelerating fields $(\varepsilon / A<0.3)$ charged $2 \pi$-kink moves in the central chain and only the radiation is observed in the neighboring chains. In higher accelerating fields $(\varepsilon / A>0.3)$ fractional charge creation is observed (Fig. 2b); it occurs in the central and in neighboring chains simultaneously, which explains the enhancement of the threshold field.

Figures $2 \mathrm{c}$ and $2 \mathrm{~d}$ show the solutions of Eqs. (13) in decelerating fields. The dynamics in weak decelerating fields $(|\varepsilon / A|<0.15)$ is analogous to the corresponding result for a single chain. The initial profile, which moves opposite the field, loses its velocity after some time and localizes near 

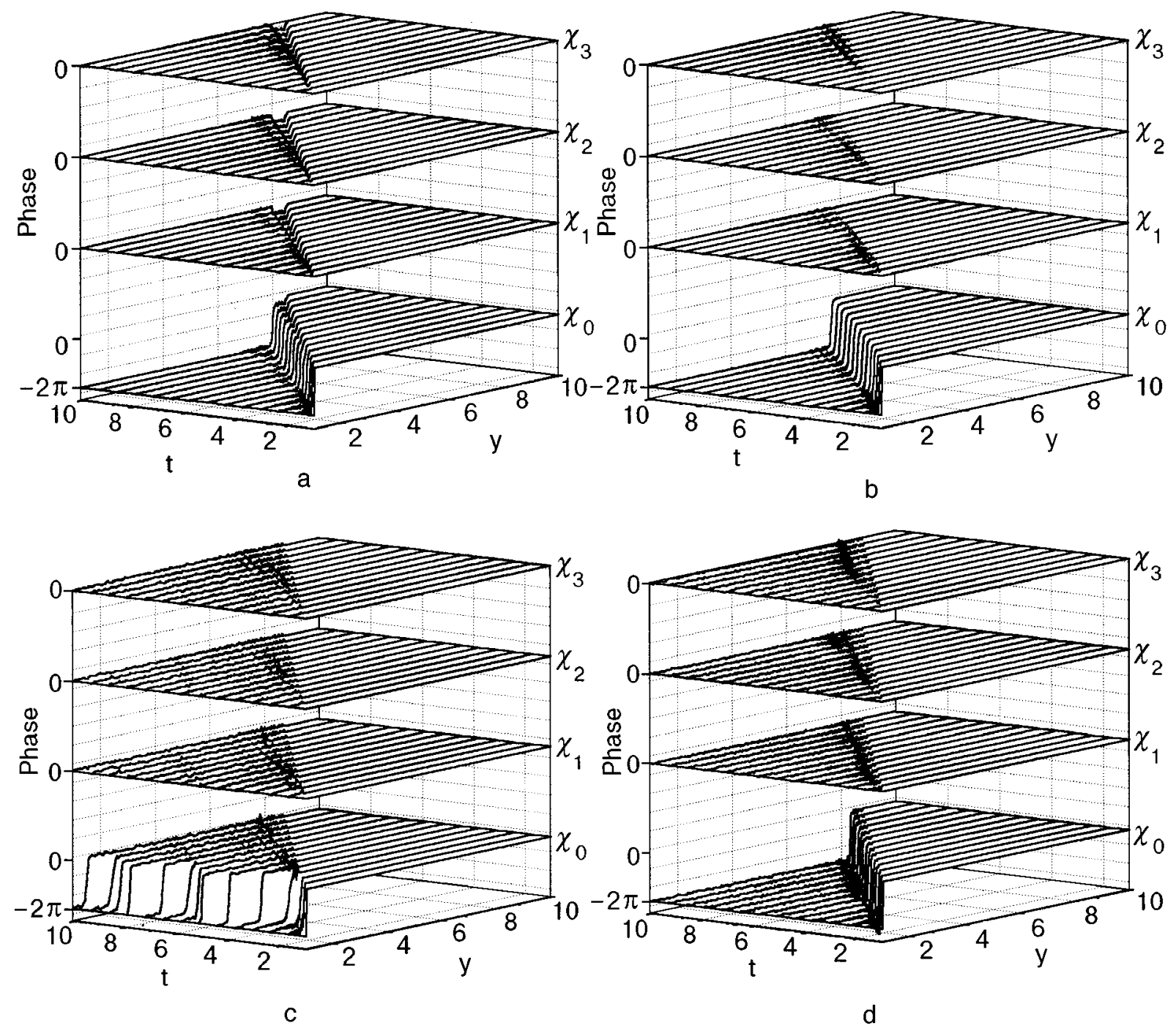

FIG. 3. Dynamics of conversion of the electrons to the $2 D$-array of chains. $B=1$ : a-incommensurate chains: $\varepsilon=0, A=0$. The initial condition transforms to a $2 \pi$-kink surrounded by dipoles; $\mathrm{b}-$ commensurate chains: $\varepsilon=0, A=1$. Commensurability leads to the suppression of the dipoles; $\mathrm{c}-\mathrm{commensurate}$ chains, decelerating field: $\varepsilon=-0.5, A=1$. The initial condition transforms to a $2 \pi$-kink decelerating by the field; d-commensurate chains, accelerating field: $\varepsilon=0.5, A=1$. The initial condition transforms to a $2 \pi$-kink accelerating by the field.

$x=0$. In higher fields $(|\varepsilon / A|>0.15)$ the charge creation is observed (Figs. $2 \mathrm{c}$ and $2 \mathrm{~d}$ ). The charge creation occurs in the neighboring chains only (Fig. 2c) or in the central and in the neighboring chains simultaneously (Fig. 2d), but always solitons and antisolitons in the neighboring chains form bound states with solitons and antisolitons in the central chain. It must be emphasized that the value of the threshold field depends on the sign of the applied field. In our opinion, this is artifact of the model; actually, in real experiment one has a symmetric system with two metal/CDW interfaces, and $E_{T}$ is independent on the sign of $\varepsilon$.

\section{BEYOND THE CLUSTER APPROXIMATION}

Consider the finite number of chains arranged symmetrically relative to the central chain. We start from the model without electric field and commensurability term (Fig. 3a). The picture obtained is in its common features similar to Fig. $2 \mathrm{a}$. The charged $2 \pi$-kink moves in the central chain and is surrounded by dipoles and radiation in the array of chains.
With increase in the size of array, the dipoles will be spread in the direction perpendicular to $x$. The amplitude of radiation is approximately the same in all the chains.

Figure $3 \mathrm{~b}$ shows the solutions of Eq. (5) at $\varepsilon=0$. The commensurability suppresses the charged dipoles. The $2 \pi$ kink moves in the central chain, losing its velocity.

In the presence of an electric field (Figs. 3c and 3d), effects of the soliton-antisoliton pairs creation and fractional charge solitons (3) are not observed. In the decelerating field (Fig. 3c) the $2 \pi$-kink looses velocity and localizes near $x=0$. In the accelerating field (Fig. $3 \mathrm{~d}$ ), the $2 \pi$-kink accompanied by radiation moves into the bulk.

In Fig. 4 the soliton-soliton interaction for the solitons located in the neighboring chains is studied. This problem is interesting in the context of the problem of the solitons aggregation into the macroscopic phase-slip centers. The $2 \pi$ kink localized in the center of the first chain is prepared in the following way: the initial condition for this chain is chosen in the form: 


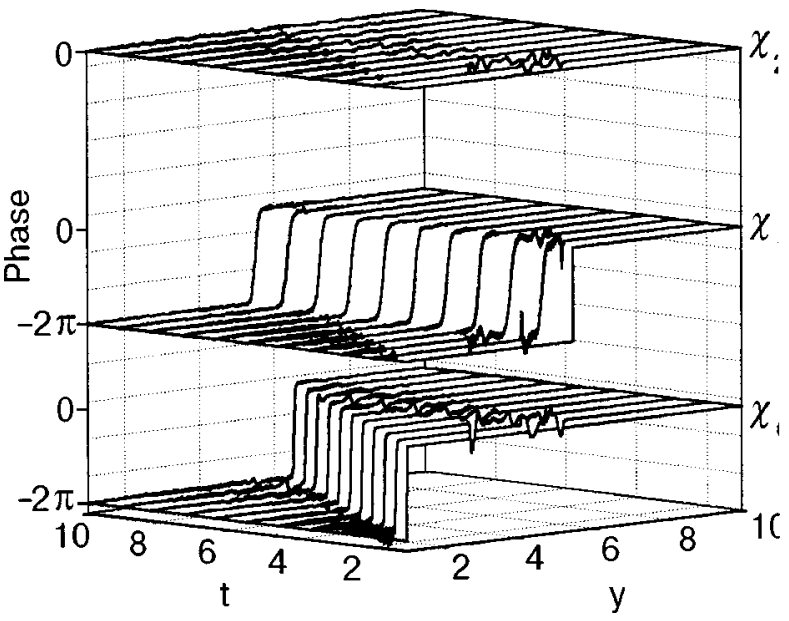

FIG. 4. Interaction between the solitons in the neighboring chains. $\varepsilon=0$, $A=B=1$. The soliton-soliton interaction leads only to small phase shift of the solitons.

$$
\varphi_{1}(t=0)=-2 \pi \theta\left(\frac{l}{2}-y\right),
$$

where $l$ is the length of the chain. Then, after some time, the condition (14) decays into the stable $2 \pi$-kink accompanied by the radiation, which spreads in both directions in all the chains. The velocity of this kink is zero due to the symmetrical initial condition (14) contrary to the initial condition (9b) which produces the kink with a nonzero velocity. Before the collision we put the radiation equal to zero. Hence, we have the static $2 \pi$-kink in the first chain and the moving $2 \pi$-kink in the zero chain. From Fig. $1 \mathrm{~b}$ we see that the velocity of the kinks does not change after collision. The collision results only in the small space shift of the kinks, which means that the aggregation of such solitons into macroscopic phase-slip centers does not occur in this model.

\section{CONCLUSIONS}

In this paper we have studied several models describing the dynamics of conversion of conduction electrons into topological solitons of the commensurate charge density wave.
In an incommensurate CDW, in the absence of electric field, the initial condition transforms into the $2 \pi$-kink localized in the central chain and surrounded by charged dipoles in the other chains. The commensurability leads to suppression of such dipoles. In the presence of electric field when $B / A \ll 1$ and the field being sufficiently large, the mechanism of topological charge creation is observed. If $B / A<1$ (which is more realistic) the fractional charge conductivity and creation of the soliton-antisoliton pairs are suppressed. The $2 \pi$ kink is localized in the central chain and is surrounded by the radiation in the other chains. The soliton velocity depends on the field.

In the framework of this model the soliton-soliton interaction does not lead to the aggregation of the solitons into macroscopic phase-slip centers.

*E-mail: pershin@ @otmail.com

${ }^{1}$ Electronic Properties of Inorganic Quasi One-Dimensional Compounds, P. Monceau (Ed.), Riedel Company, Dordrecht (1985), Parts 1 and 2, Density Waves in Solids, G. Grüner (Ed.), Addison-Wesley, Reading, Massachusets (1994).

${ }^{2}$ I. V. Krive, A. S. Rozhavsky, and I. O. Kulik, Fiz. Nizk Temp. 12, 635 (1986) [ Sov. J. Low Temp. Phys. 12, 360 (1986)]

${ }^{3}$ S. Brazovskii and N. Kirova, Electron Self-Localization and Periodic Superstructures in Quasi One Dimensional Dielectrics, Sov. Sci. Rev. A: Phys., I. M. Khalatnikov (Ed.), Harwood Academic Publishers, 6 (1984).

${ }^{4}$ S. Brazovskii and S. Matveenko, J. Phys. I (Paris) 1, 269 (1991); J. Phys.

I (Paris) 1, 1173 (1991).

${ }^{5}$ D. Feinberg and J. Friedel, J. Phys. (Paris) 49, 485 (1988).

${ }^{6}$ J. C. Gill, J. Phys. C 19, 6589 (1986).

${ }^{7}$ S. Ramakrishna, M. P. Maher, V. Ambegaokar, and U. Eckern, Phys. Rev. Lett. 68, 2066 (1992)

${ }^{8}$ A. S. Kovalev, Yu. V. Pershin, and A. S. Rozhavsky, Phys. Rev. B 53, 16227 (1996); Fiz. Nizk. Temp. 22, 322 (1996) [Low Temp. Phys. 22, 253 (1996)].

${ }^{9}$ I. V. Krive, A. S. Rozhavsky, and V. A. Rubakov, Pis'ma Zh. Éksp. Teor. Fiz 46, 99 (1987) [JETP Lett. 46, 121 (1987)].

${ }^{10}$ A. S. Rozhavsky, Yu. V. Pershin, and A. S. Kovalev, Fiz. Nizk. Temp. 23, 1325 (1997) [Low Temp. Phys. 23, 996 (1997)].

${ }^{11}$ Yu. S. Kivshar and A. M. Kosevich, Pis'ma Zh. Éksp. Teor. Fiz 37, 542 (1983) [JETP Lett. 37, 648 (1983)].

${ }^{12}$ I. Ishimori and T. Munakata, J. Appl. Phys. 51, 3367 (1982).

This article was published in English in the original Russian journal. It was edited by R. T. Beyer. 